\title{
Comparative Shape and Electrostatic Study of Highly Potent and Selective CYP1B1 Inhibitor: Assessment of Active Site of CYP1B1 by Binding Mode Analysis Using Site Map Tool
}

\author{
Mohd Usman Mohd Siddique, Barij Nayan Sinha, Venkatesan Jayaprakash* \\ Department of Pharmaceutical Sciences and Technology, Birla Institute of Technology, Mesra, Ranchi, INDIA.
}

\begin{abstract}
Introduction: The major aim of drug design and discovery is to minimize the time and cost of drug discovery process. Various molecules which are promised to be potential candidate during computational and preclinical studies, shows the poor results during clinical trials due to less credibility of in silico results. This leads to increased burden of time and cost of drug discovery process. Methodology: A reliabel Shape and Electrostatic similarity based screening of ligands and assessment of druggability of the target protein provides a means to predict the negatives at an earlier stage of drug discovery pipeline. Two compounds (BNUA-3 \& BNUB-13) reported from our lab were compared with ANF and TMS. Results and Discussion: Shape coefficient between BNUB-13 and TMS was 0.79 and electrostatic coefficient was 0.464 indicating that BNUB-13 is quite similar to TMS. Dscore values for ANF, TMS, BNUB-13 and BNAU-3 were also found to be similar, $1.404,1.390,1.389$ and 1.366, respectively. Conclusion: The comparative studies of two highly potent CYP1B1 inhibitors revealed minimum structural information that can modulate the potency of the inhibitors. Meanwhile assessment of the active site of CYP1B1 has shown that CYP1B1 is a druggable target.
\end{abstract}

Key words: CYP1B1, Shape electrostatic coefficient, Sitemap analysis, Druggability, In silico ADME calculation.

\section{INTRODUCTION}

Cytochrome P450 (CYP) enzymes are present in various organs of the human body that comprises a Formatting: leave a space here large family of detoxification enzymes. The cytochrome P450 1B1 isoform (CYP1B1) is a heme-thiolate monooxygenase which causes the hydroxylation of steroids, estrogens and fatty acids. Unlike other CYPs, CYP1B1 is not present in normal healthy tissues but significantly expressed in cancerous cells of hormonal cancers including that of the ovary, prostate, uterus, mammary, pituitary, regardless of the cancer's genetic origin. Recent studies revealed that CYP1B1 plays a major role in the genesis of hormone-mediated prostate and breast cancers. ${ }^{1,2,3}$ In both cancerous and precancerous cells of mammary, prostate and ovarian tissues, the regiospecific metabolism of estradiol produces '4-hydroxy estradiol (4-OHE 2 )' by CYP1B1 that was reported to be one of the major reason for oncogenesis. ${ }^{4}$ In cancer cells these metabolite further oxidized to 3, 4-estradiol quinone that forms depurinating adducts with DNA and tubuline leading to genotoxic mutations. $5,6,7,8,9,10,11,12$ Therefore, the rate and extent of CYP1B1 expression in endomatrium, mammary and ovarian tissues can be considered as potential biomarker for hormonal oncogenesis. $2,5,13,14,15$

Recently we reported two series of compounds with different chemical scaffold with potent and selective inhibitory activity on CYP1B1. ${ }^{16,17}$ This has suggested that
Submission Date: 21-07-2017; Revision Date: 25-09-2017; Accepted Date: 10-01-2018

DOI: 10.5530/ijper.52.1.18 Correspondence:

Dr. Venkatesan Jayaprakash,

Department of Pharmaceutical Sciences and Technology, Birla Institute of Technology Mesra, Ranchi, INDIA.

Phone no: +91 9470137264; Email: drvenkatesanj@gmail. com

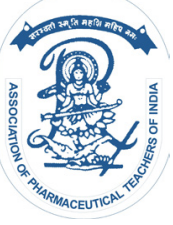

www.ijper.org 


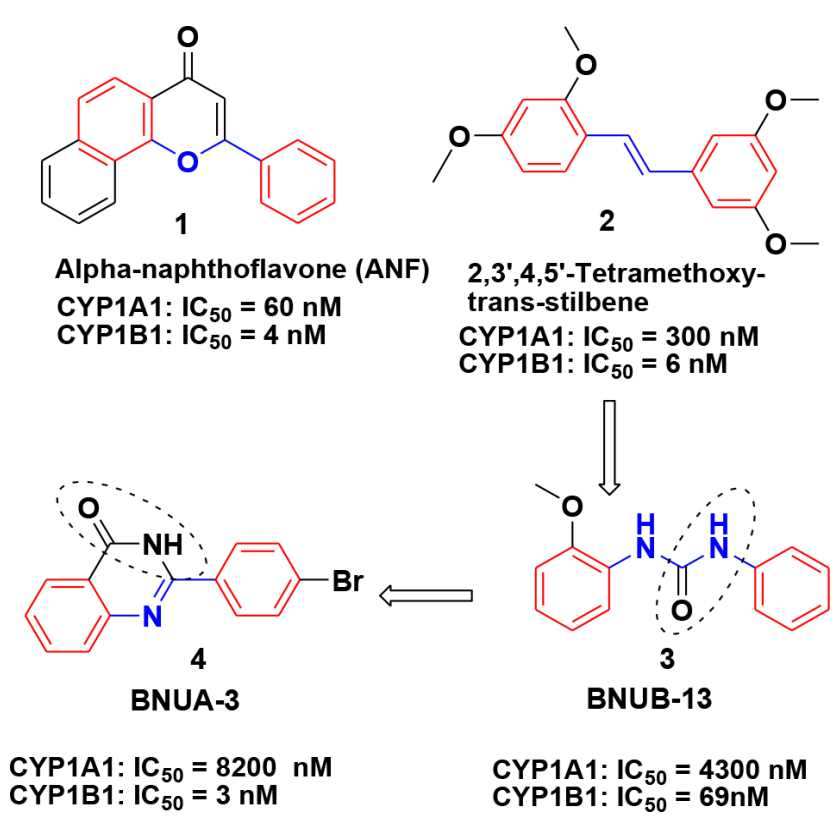

Figure 1: Potent CYP1B1 inhibitors used for druggability predictions.

some molecular properties are similar for these two chemical scaffolds in terms of their interaction with the target protein. The current investigation is aimed at unraveling them with a series of computational techniques that may help in designing CYP1B1 selective inhibitors.

Active sites of CYP1 family enzymes have $\leq 40 \%$ of amino acid sequence homology and CYP1A1 and CYP1A2 shares $\geq 55 \%$ sequence homology. So it is quite difficult to design a highly specific and selective inhibitor against these iso-enzymes. ${ }^{18,19}$ Several attempts have been reported on the development of computational models with the intention of predicting enhanced potency and selectivity of inhibitors. Steric and electrostatic properties of the molecules are the two most important parameters in predicting the binding affinity and selectivity towards the target proteins. These studies provide useful information for future drug design efforts. Comparative Molecular Field Analysis (CoMFA) and Comparative Molecular Similarity Indices Analysis (CoMSIA) were employed to study the structure activity relationship of a series of molecules having same chemical scaffold by overlaying the $3 \mathrm{D}$ structures of ligands. But these methods have a limitation in the absence of protein that interacts with them in biospace. Molecular dynamic (MD) simulations based methods were recently described for studying ligand-protein interactions at molecular level. ${ }^{20,21,22}$ In the current study we employed Shape and Electrostatic similarity assessment, ${ }^{23}$ of two highly specific and potent CYP1B1 inhibitors TMS and BNUB-13. Moreover binding site was also evaluated by site map, which is novel and effective algorithm for accurate and rapidactive site identification. We also assessed the druggability of active site pocket of CYP1B1 (PDB ID: 3PM0) ${ }^{24}$ by comparing the DScore values of four known and potent inhibitors of CYP1B1, Tetramethoxy stilbene (TMS), ${ }^{25}$ alphanaphthafalvone (ANF) ${ }^{26,27}$ BNUB-13 ${ }^{16}$ and BNUA $-3^{17}$ Thus we believe this study will provide useful insight in designing selective and potent CYP1B1 inhibitors.

\section{MATERIAL AND METHODS}

Shape and electrostatic analysis was performed by using VROCS (3.1.2) and EON (2.1.0) of OpenEye toolkit. ${ }^{28}$ using TMS (reference molecule) and BNUB-13 (test molecule). Molecular docking and Site map analysis were done on Maestro 8.5. and SiteMap module of Schrodinger LLC suite. ${ }^{29}$ respectively, running on RHEL5 operating system installed on DELL Precision T3400 machine (n-series, Intel core 2 Quad processor, 8GB RAM, 500GB). Both molecules were prepared in Glide and energy minimized using ligprep module implemented in Maestro 8.5.111. The co-crystal structure of CYP1B1 (PDB Code: 3PM0) was downloaded from www.rcsb.org. For validation of software the internal ligand was extracted and redocked into the active site. Protein was prepared for docking through Protein preparation wizard and Grid was generated through grid preparation wizard picking ligand to specify the binding site. Docking was performed using GLIDE 5.0 with XP protocol. The docked conformers were analyzed through XP-visualizer. Default parameters were employed during all the computational studies.

\section{Shape and Electrostatic Study}

Shape and electrostatic comparative study was done because it gives an idea about physicochemical aspects of molecular recognition. Thus comparing shape complementary and electronic features of our molecule with BNUB-13 has revealed important structural framework which realized the binding affinity, which in turn functional potency (Figure 1). For these we employed the ROCS algorithm (Rapid Overlay of Chemical Structures) and EON of OpenEye Toolkit. During the round of ROCS analysis BNUB-13 and TMS were superimposed based on their shape agreement. Then they were subjected to electrostatic correlations using EON and results were analyzed based on their Shape and Electrostatic Tanimoto as shown in Figure 2.

Binding site of CYP1B1 was evaluated by Sitemap tool of Schrödinger. All the molecules were separately tested for calculation of Dscore, hydorphilicty, hydrophobicity, $\mathrm{H}$ bond donor and acceptor properties. These com- 
puted properties are used to calculate the site score and Dscore. Depending on these scores, the druggability of target was also predicted.

\section{In-silico ADME prediction}

Pharmacokinetic properties (ADME Absorption, Distribution, Metabolism and Excretion) are the crucial part of new drug development procedure as many molecules are withdrawn from the market due to their poor pharmacokinetic profiles. We used QikProp, version 3.0, Schrödinger, LLC, New York, 2005, for prediction of ADME properties. To calculate drug likeliness properties we consider various parameters such as molecular weight, $\mathrm{H}$ bond donor, $\mathrm{H}$ bond acceptor, polar surface area and predicted aqueous solubility and human oral absorption.

\section{RESULTS AND DISCUSSION}

\section{Shape and electrostatic studies}

The rational in comparing shape and electrostatic properties is that it is primary topological determinants of molecule, which can be considered for better fitness and binding in the active site pocket of protein or enzyme. Recently Bostrom et al, reported the discovery of potent fibrinolysis inhibitor by Shape and Electrostatic Complementarity to the Drug Tranexamic Acid which has the potential for the treatment of bleeding disorders. ${ }^{23}$ By the comparative shape and electrostatic study we got good correlation between TMS and BNUB-13. Shape coefficient between two molecules was 0.769 while electrostatic coefficient was found to be 0.464 indicating that BNUB-13 was quite similar to TMS (Figure 3). Owing to the structural simiarity, the newly iden-
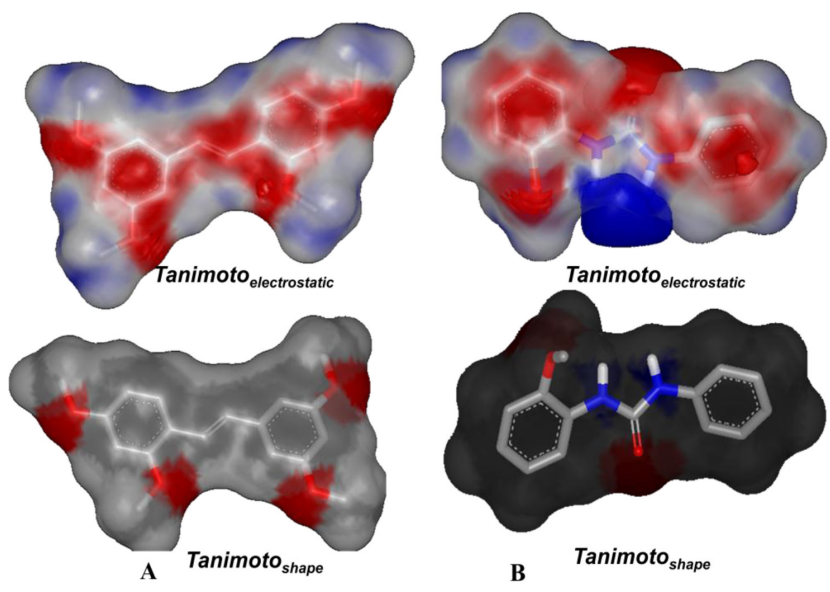

Figure 2: Predicted three-dimensional shape and Tanimoto overlays of compounds TMS (A) and BNUB-13 (B)..$^{30}$ tified molecule could efficiently fit into the active site pocket of CYP1B1.

TMS and ANF are non-selective CYP inhibitors but when the non-polar bridge is substituted by a polar urea linker as in biphenyl urea the specificity of molecule was found to increase. It has been observed in BNUB13 with $\mathrm{IC}_{50}$ value of $69 \mathrm{nM}$ and specificity of 62-fold. Hybridizing the structures of ANF and BNUB-13 we got new scaffold which maintained its planarity and the cyclized urea linker resembled ANF. Surprisingly the resultant molecule was highly specific and emerged as the most potent inhibitor (BNUA-3) with $\mathrm{IC}_{50}$ value of $3 \mathrm{nM}$. In-scilico and in-vitro results were in coherence with the important structural features that would guide the design and discovery of potent and specific CYP1B1 inhibitor.

\section{Molecular modeling studies}

The X-ray crystallographic studies reveal that CYP1B1 has the rounder shape narrow slot-like substrate binding cavity, which gets occupied by ANF and hinder the interactions between heme iron-oxo intermediate and planner substrate. ${ }^{24}$ Molecular docking studies revealed the interactions between the terminal aromatic carbons of BNUB-13 and reactive heme iron-oxo via van der Waals' interactions (distance is $<5^{\circ} \mathrm{A}$ ). The second aromatic ring of urea moiety positioned in such a way that it shows the $\pi-\pi$ interactions with hydrophobic
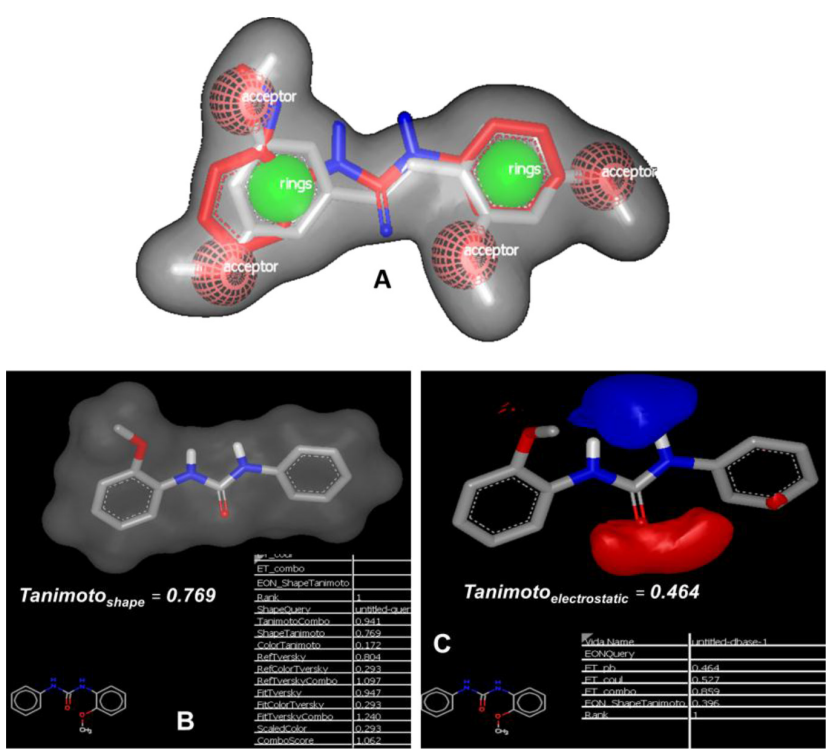

Figure 3: (A) Complete overlapping of TMS (silver color) and BNUB-13 (red color); (B) the display of shape and (C) electrostatic potentials for BNUB-13 with that of TMS in their neutral form. Blue color denotes electropositive areas, whereas red color shows electronegative areas. The calculated Tanimoto values depicted that BNUB-13 is electrostatically very similar to TMS. 
Phe231 and Phe268 residues of CYP1B1. In addition, BNUB-13 also display the polar interactions via H-bonding with Asp333 (Figure 4C). Overall, these interactions of the urea derivative with CYP1B1 contribute the potent inhibition of CYP1B1 at low nanomolar $\mathrm{IC}_{50}$ value.

Similarly aromatic ring $\mathrm{C}$ of BNAU-3 flanks towards the heme atom due to presence of heteroatom containing ring $\mathrm{B}$ and interacts with heme via van der Waals interactions (distance is $<5^{\circ} \mathrm{A}$ ) in a way similar to the benzo[a]chromane ring of ANF. The geometry of BNAU-3 facilitates interaction in such a way that $\mathrm{O}$ atom of ring $\mathrm{C}$ comes nearest to iron cofactor of heme. Moreover ring $\mathrm{A}$ and $\mathrm{B}$ display hydrophobic interaction with Phe231. Additionally BNAU-3 also interacts with Phe134 residue via $\pi-\pi$ interactions. These interactions with CYP1B1 make the compound more potent inhibitor of CYP1B1. All molecules occupied the rounder shape narrow slot-like substrate binding cavity and interact with the Phe231 and Phe268, and one aromatic ring interact with heme moiety. But in case of BNAU3 , it has additional polar $\mathrm{H}$ bonding with Asp333 that make this molecule more selective towards the CYP1B1 isoform. Similarly in case of BNUB-13 there is additional hydrophobic interaction with Phe134 residue and position of $\mathrm{C}$ ring $\mathrm{O}$ atom is very close to iron of heme moiety leading to most potent and selective inhibition.

\section{Characterization of CYP1B1 active site as druggable target}

Site map tool was used to find out whether a protein can be a druggable target or not. For validation of druggability of active site of CYP1B1 enzyme (PDB ID: 3PM0) we used four potent CYP1B1 inhibitor ANF (50 nM), TMS (6 nM), BNUB-13 (69 $\mathrm{nM})$ and BNUA-3 $(3 \mathrm{nM})$. The results were summarized in Table 1. Dscore as reported earlier, it is calculated based on the sitescore, size, hydrophilicity, hydrophobicity, enclosure, exposure and $\mathrm{H}$ bond donor and acceptor values. ${ }^{31}$ The Figure 5 (A) comprise of the ANF binding site map in active site pocket of CYP1B1 while. The calculation is based on the hydrophobicity of the pocket for the druggability and expresses in terms of numerical values $0-2$. Highly hydrophilic cavities are considered as undruggable target or protein and have the value of 0 , while 1 is considered difficult to target and 2 falls in druggable target category. Site map analysis for complexes of all the four ligands with CYP1B1 was performed to check the druggability of the target protein. The calculation using all the four complexes displayed that the protein is druggable because all molecules showed D-score that expressed them
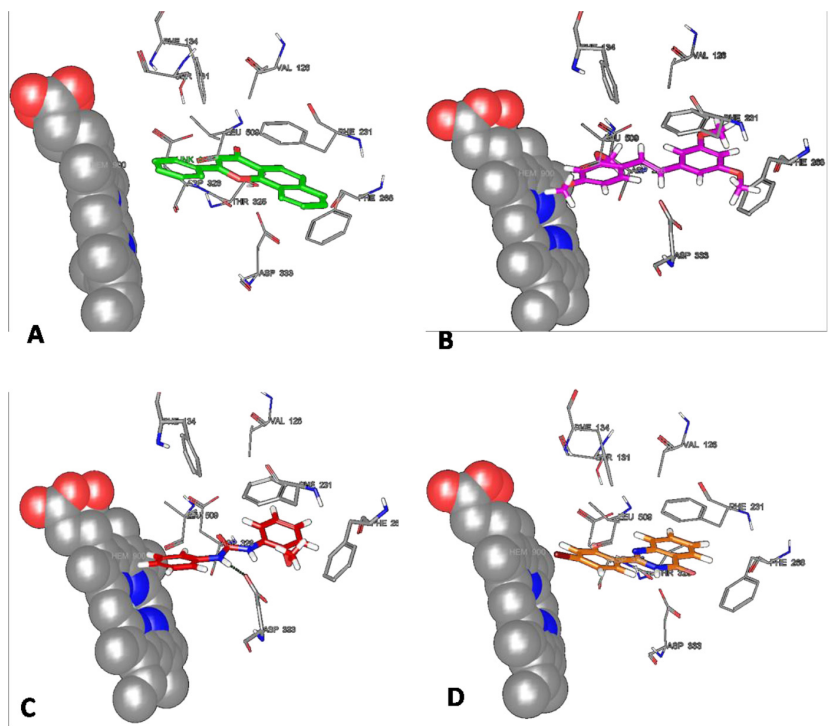

Figure 4: 3D interaction diagram of (A) ANF, (B) TMS (C) BNUB-13 and (D) BNUA-3 at active site of CYP1B1 enzyme and showing the interactions with amino acid residues present at the active site (PDB ID: 3PM0).
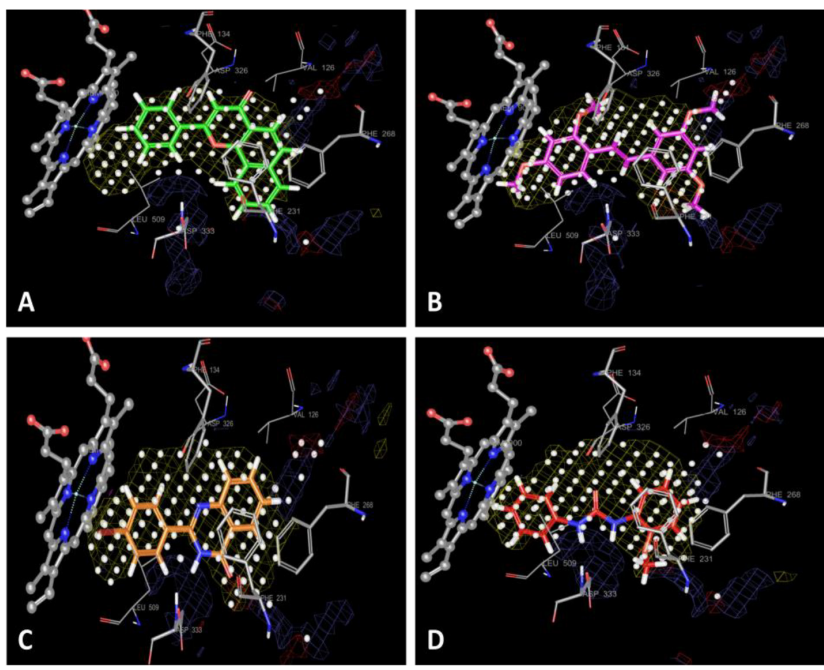

Figure 5: Binding mode analysis of ANF (A), TMS (B), BNUA-3 (C) and (D) BNUB-13. The various crucial interactions are red color denotes $\mathrm{H}$ Bond acceptor, blue color shows H-Bond donor, Yellow color indicates hydrophobic interaction and green color represents hydrophilic interaction.

as druggable site score 2 (Table 1). The hydrophobic score of ANF was found be better than the other three. BNUB-13 having the better hydrophobic interaction than BNUA-3, but it is less potent than BNUA-3 due to its polar bridge (higher hydrophilic score). We suppose that the compounds having less polar bridges perform well in terms of improving potency. All physicochemical properties obtained were identical in all molecules (Table 1). 


\begin{tabular}{|c|c|c|c|c|c|c|c|c|c|}
\hline \multicolumn{8}{|c|}{ Table 1: SiteMap Property Values and Dscore Ranks for the potent CYP1B1 inhibitors. } \\
\hline Entry & $\begin{array}{c}\text { IC }_{50} \text { nm } \\
\text { (CYP1B1) }\end{array}$ & Category & DScore & SiteScore & Size & Enclosure & Exposure & hydorphilic & hydrophobic \\
\hline ANF & $50^{\mathrm{a}}$ & 2 & 1.400 & 1.310 & 117 & 0.971 & 0.316 & 0.377 & 5.044 \\
\hline TMS & 6 & 2 & 1.390 & 1.305 & 114 & 0.971 & 0.349 & 0.406 & 4.836 \\
\hline BNUB-13 & $69^{a}$ & 2 & 1.389 & 1.304 & 116 & 0.972 & 0.288 & 0.412 & 4.874 \\
\hline BNUA-3 & $3^{\text {a }}$ & 2 & 1.366 & 1.290 & 124 & 0.970 & 0.337 & 0.477 & 4.648 \\
\hline
\end{tabular}

${ }^{\mathrm{a}} \mathrm{IC}_{50}$ values were determined in Sacchrosomes ${ }^{\mathrm{TM}}$ and it represents mean and standard deviations from three independent experiments

\begin{tabular}{|c|c|c|c|c|c|c|c|c|}
\hline Entry & $\begin{array}{c}\text { Lipinski Rule } \\
\text { Of Five }\end{array}$ & Mol Wt & $\begin{array}{c}\text { PLog P } \\
(\mathrm{o} / \mathrm{w})^{\mathrm{a}}\end{array}$ & PSA $^{b}$ & HBA $^{c}$ & $H_{B D}^{d}$ & plogBBe & $\begin{array}{c}\% \text { Oral } \\
\text { absorption }\end{array}$ \\
\hline ANF & 0 & 272.303 & 4.141 & 33.670 & 2.5 & 1 & 0.081 & 100 \\
\hline TMS & 0 & 300.354 & 4.094 & 32.816 & 3 & 0 & -0.443 & 100 \\
\hline BNUB-13 & 0 & 242.277 & 4.016 & 52.670 & 2.750 & 2 & -0.090 & 100 \\
\hline BNUA-3 & 0 & 301.342 & 3.070 & 50.152 & 3.5 & 1. & -0.143 & 100 \\
\hline
\end{tabular}

a Predicted octanol/water partition coefficient.

b Polar surface area.

' Hydrogen bond acceptor.

${ }^{\mathrm{d}}$ Hydrogen bond donor.

e Predicted brain/blood partition coefficient (-3-1.2).

\section{In silico ADME prediction}

In-silico ADMET calculation was performed for drug likeness properties of molecules. The descriptors like dipole moment, the percentage of oral absorption, and $\mathrm{P} \log \mathrm{BB}$ were analysed. Lipinski's Rule of 5 (Ro5) explains, in orally active molecules hydrogen bond acceptor (HBA) should not exceeds more than 10 and molecular weight (MW) 500D. Whereas $\mathrm{H}$ bond donor (HBD) groups less than 5 and it should have oil/water partition coefficient $(\log \mathrm{Po} / \mathrm{w})$ below 5 .The results thus obtained were presented in Table 2. All the molecules were found to comply with the rule-of-five (R05) described by Lipinski. The CNS toxicity was predicted by blood-brain coefficient (plogBB), and the results indicate that all molecules have values within the range and suggest that none of the molecules can cross the blood brain barrier to produce CNS toxicity.

\section{CONCLUSION}

Physicochemical properties of ligand and protein active site are important in drug design and discovery and protein can be called druggable if it can exhibit in vivo pharmacological response by drug like molecule. Shape and electrostatic parameter determine the physicochemical properties of any molecule. Comparing and calculation of these parameters of known potent inhibitors would give important ideas that could be taken into consideration while designing of any new molecule against particular target or protein. Moreover accurate prediction of druggability of CYP1B1 generate potential 3Dstructuralinformation of receptorgivingvitalinformation. Having this information of whethera given protein is druggable or not at an early stage of drug discovery therefore has the potential of saving considerable time and expense. In silico ADMET calculations showed that all the molecules complied with Lipinski rule of five and hence have the highest probability to become drug (drug-likeness).

\section{CONFLICT OF INTEREST}

Authors do not have any conflict of interest

\section{ACKNOWLEDGMENT}

MUMS acknowledge UGC for award of MANF JRF fellowship (201516-MANF-2015-17-MAH-60712).

\section{ABBREVIATIONS USED}

ANF: Alphanaphthaflavone; CYPs: Cytochrome P450; CYP1B1: Cytochrome P450-1B1; TMS: Tetramethoxy stilbene; ROCS: Rapid Overlay of Chemical Structures.

\section{REFERENCES}

1. McFadyen MC, Breeman S, Payne S, Stirk C, Miller ID, Melvin WT, et al. Immunohistochemical localization of cytochrome P450 CYP1B1 in breast cancer with monoclonal antibodies specific for CYP1B1. Journal of Histochemistry andCytochemistry. 1999; 47(11):1457-64. 
2. Murray GI, Taylor MC, McFadye MC, McKay JA, Greenlee WF, Burke MD, et al. Tumor-specific expression of cytochrome P450 CYP1B1. Cancer Research. 1997;57 (14):3026-31.

3. Muskhelishvili L, Thompson PA, Kusewitt DF, Wang C, Kadlubar FF. In situ hybridization and immunohistochemical analysis of cytochrome P450 1B1 expression in human normal tissues. Journal of Histochemistry and Cytochemistry. 2001;49(2):229-36.

4. Yager JD, Endogenous estrogens as carcinogens through metabolic activation. Journal of National Cancer Institute Monographs. 2000;27(27):6773.

5. Cavalieri EL, Devanesan P, Bosland MC, Badawi AF, Rogan EG. Catechol estrogen metabolites and conjugates in different regions of the prostate of Noble rats treated with 4-hydroxyestradiol: implications for estrogen-induced initiation of prostate cancer. Carcinogenesis. 2002;23(2):329-33.

6. Cavalieri E, Stack D, Devanesan P, Todorovic R, Dwivedy I, Higginbotham S, et al. Molecular origin of cancer: catechol estrogen-3, 4-quinones as endogenous tumor initiators. Proceedings of the National Academy of Sciences. 1997;94(20):10937-42

7. Hayes CL, Spink DC, Spink BC, Cao JQ, Walker N J, Sutter TR. 17 beta-estradiol hydroxylation catalyzed by human cytochrome P450 $1 \mathrm{~B} 1$. Proceedings of the National Academy of Sciences. 1996;93(18):9776-81.

8. Rogan EG, Badawi AF, Devanesan PD, Meza JL, Edney JA, West WW. Higginbotham SM, Cavalieri EL. Relative imbalances in estrogen metabolism and conjugation in breast tissue of women with carcinoma: potential biomarkers of susceptibility to cancer. Carcinogenesis. 2003;24(4):697-702.

9. Zhu BT, Conney AH. Functional role of estrogen metabolism in target cells: review and perspectives. Carcinogenesis. 1998;19(1):1-27.

10. Rochat B, Morsman, JM, Murray GI, Figg WD, McLeod HL. Human CYP1B1 and anticancer agent metabolism: mechanism for tumor-specific drug inactivation? Journal of Pharmacology and Experimental Therapeutics. 2001;296(2):537-41.

11. Newbold RR, Liehr JG. Induction of uterine adenocarcinoma in CD-1 mice by catechol estrogens. Cancer Research. 2000;60(2):235-7.

12. Cavalieri EL, Rogan EG. A unifying mechanism in the initiation of cancer and other diseases by catechol quinones. Annals of the New York Academy of Sciences. 2004; 1028(1):247-57.

13. Liehr JG, Ricci MJ. 4-Hydroxylation of estrogens as marker of human mammary tumors. Proceedings of the National Academy of Sciences 1996;93(8):3294-6.

14. Spink DC, Spink BC, Cao JQ, DePasquale JA, Pentecost BT, Fasco MJ, et al. Differential expression of CYP1A1 and CYP1B1 in human breast epithelial cells and breast tumor cells. Carcinogenesis. 1998;19(2):291-8.

15. Tait L, Soule HD, Russo J. Ultrastructural and immunocytochemical characterization of an immortalized human breast epithelial cell line, MCF-10. Cancer Research. 1990;50(18):6087-94.
16. Siddique MUM, McCann GJ, Sonawane V, Horley N, Williams IS, Joshi P et al. Biphenyl urea derivatives as selective CYP1B1 inhibitors. Organic andBiomolecular Chemistry. 2016;14(38):8931-6.

17. Siddique MUM, McCann GJ, Sonawane VR, Horley N, Gatchie L, Joshi P, et al. Quinazoline derivatives as selective CYP1B1 inhibitors. European Journal of Medicinal Chemistry. 2017;130:320-7.

18. Nebert DW, Nelson DR, Adesnik M, Coon MJ, Estabrook RW, Gonzalez FJ, et al. The p450 superfamily: updated listing of all genes and recommended nomenclature for the chromosomal loci. DNA. 1989:8(1):1-13.

19. Testa B, Caldwell J. Monooxygen ase-catalyzed NC cleavage. The metabolism of drugs and other xenobiotics - biochemistry of Redox reactions. Testa B, Caldwell J (eds), Academic Press limited, London 1995;215-24.

20. Guvench O, MacKerell Jr AD. Computational fragment-based binding site identification by ligand competitive saturation. PLoS Computational Biology. 2009;5(7):e1000435.

21. Raman EP, Yu W, Guvench O, MacKerell Jr AD. Reproducing crystal binding modes of ligand functional groups using Site-Identification by Ligand Competitive Saturation (SILCS) simulations. Journal of Chemical Information and Modeling. 2011;51(4):877-96.

22. Seco J, Luque FJ, Barril X. Binding site detection and druggability index from first principles. Journal of Medicianal Chemitry. 2009;52(8):2363-71.

23. Boström J, Grant JA, Fjellström O, Thelin A, Gustafsson D. Potent fibrinolysis inhibitor discovered by shape and electrostatic complementarity to the drug tranexamic acid. Journal of Medicinal Chemistry. 2013;56(8):3273-80.

24. Wang A, Savas U, Stout CD, Johnson EF. Structural characterization of the complex between a-naphthoflavone and human cytochrome P450 1B1. Journal of Biological Chemistry. 2011;286(7):5736-43.

25. Kim S, Ko H, Park JE, Jung S, Lee SK, Chun YJ. Design, synthesis, and discovery of novel trans-stilbene analogues as potent and selective human cytochrome P450 1B1 inhibitors. Journal of Medicinal Chemistry. 2002;45(1):160-4.

26. Campbell DR, Kurzer MS. Flavonoid inhibition of aromatase enzyme activity in human preadipocytes. Journal of Steroid Biochemistry and Molecular Biology. 1993:46(3):381-8.

27. Kellis Jr JT, Vickery LE. Inhibition of human estrogen synthetase (aromatase) by flavones. Science. 1984;225:1032-5.

28. OEChem T, version 2.0. 0. OpenEye Scientific Software, Santa Fe, NM. There is no corresponding record for this reference.

29. Schrodinger L. Schrodinger Software Suite. New York: Schrödinger, LLC 2011.

30. Halgren TA. Identifying and characterizing binding sites and assessing druggability. Journal of Chemical Information and Modeling. 2009;49(2):37789.

31. Cheng AC, Coleman RG, Smyth KT, Cao Q, Soulard P, Caffrey DR, et al. Structure-based maximal affinity model predicts small-molecule druggability. Nature Biotechnology. 2007;25(1):71-5.

\section{PICTORIAL ABSTRACT}

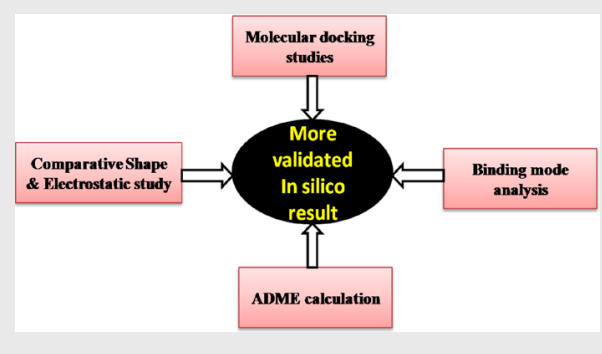

\section{SUMMARY}

The combined approach of ligand and receptor aspect were considered for generation of in silico data. Shape and electrostatic studeis reveals the structural complementary that are prerequisite for inhibitory activity.

Binding mode analysis gave the receptor 3D structural information that can be used for lead optimization in drug discovery, to modify the ligand to enhance its binding affinity and to improve its physico-chemical properties. 


\section{About Authors}

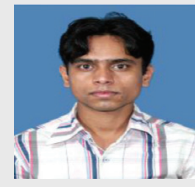

Mohd Usman Mohd Siddique: Is research scholar in the Department of Pharmaceutical Sciences andTechnology, BIT Mesra Ranchi. He has the area of interest in design and discovery of novel molecules against cancer and viral infection. He has completed his M S (Pharm) degree from National Institute of Pharmaceutical Education and Research Kolkata.

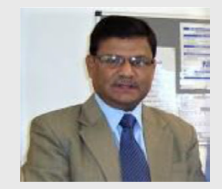

Dr B.N. Sinha is Professor andFormer Head at the Department of Pharmaceutical Sciences andTechnology, Birla Institute of Technology, Mesra. He obtained his B. Pharm. from BIT Mesra, Ranchi in 1981 followed by M. Pharm. from Banaras Hindu University in 1983. Prof. Sinha completed his PhD from Birla Institute of Technology, Mesra in 1997. He is a medicinal chemist having research interest in the area of Drug Discovery Approaches with regard to Synthetic and Natural Leads using Modern Analytical Techniques and Soft wares.

Dr. Venkatesan Jayaprakash: Currently working as Associate Professor in Department of Pharmaceutical Sciences andTechnology, Birla Institute of Technology, Mesra. He obtained his B. Pharmacy from Madras Medical College, M. Pharmacy from Banaras Hindu University and PhD from Birla Institute of Technology, Mesra. He did his doctoral research under the supervision of Dr. B. N. Sinha. He is a medicinal chemist having expertise in organic synthesis and molecular modeling.

Cite this article: Siddique MUM, Sinha BN, Jayaprakash V. Comparative Shape and Electrostatic Study of Highly Potent and Selective CYP1B1 Inhibitor: Assessment of Active Site of CYP1B1 by Binding Mode Analysis Using Site Map Tool. Indian J of Pharmaceutical Education and Research. 2018;52(1):159-65. 